\title{
Ueber die Bildung von Ornithin bei der Spaltung des Arginin's und über die Constitution dieser beiden Basen. \\ Von
}

E. Schulze und E. Winterstein.

(Aus dem agriculturohemischen Laboratorium des Polytechnikums in Ztirich.)

(Der Redaction zugegangen am 30. Juli 1898.)

Durch den von S. G. Hedin ${ }^{1}$ ) erbrachten Nachweis, dass bei der Spaltung der Eiweissstoffe durch Salzsäure Arginin entsteht, nicht minder aber durch A. Koss el's ${ }^{2}$ ) Beobachtungen über die Bildung der gleichen Stickstoffverbindung aus den Protaminen hat die Frage nach der Constitution des Arginins an Bedeutung gewonnen. Kossel vermuthet, dass die Protamine, welche bei der Spaltung durch Säuren nur Basen, keine Amidosäuren liefern, als Kerne in den Eiweissmolekülen enthalten sind; die Aufklärung der Constitution der Protamine würde daher wohl ein bedeutsamer Schritt auf dem Wege sein, der in hoffentlich nicht allzu ferner Zeit zur Erforschung der Constitution aller Eiweisskörper führen wird. Da nun aber die Protamine bei der Spaltung weit mehr Arginin als andere Basen liefern, so lässt sich ihre Constitution niçht aufklären, ohne dass man zuvor über die Structur des Arginins sich Aufschluss verschafft hat.

E. Schulze und A. Likiernik ${ }^{3}$ ) haben nachgewiesen, dass Arginin beim Erhitzen mit Barytwasser Harnstoff liefert

1) Diese Zeitschrift, Bd. XXI, S. 155.

2) Ebendaselbst, Bd. XXII, S. 176, sowie Bd. XXV, S. 165; Sitzungsberichte der Gesellschaft zur Förderung der Naturwissenschaften in Marburg, Juliheft 1897.

3) Berichte der D. Chem. Gesellschaft, Bd. 24, S. 2701.

Hoppe-Seyler's Zeitschrift f. physiol. Chemie, XXVI. 
- eine Thatsache, welche es als möglich erscheinen lässt, dass jene Base ein Derivat des Guanidins ist. Um in der Erforschung der Constitution des Arginins weiter zu kommen, mussten wir Aufschluss über die Produkte zu gewinnen suchen, welche bei der Spaltung dieser Base durch Barytwasser neben Harnstoff entstehen. Es gelang uns, aus diesen Produkten eine stickstoffhaltige Base $\mathrm{zu}$ isoliren, welche für identisch mit dem von M. Jaffé ${ }^{1}$ ) entdeckten Ornithin $=\mathrm{C}_{3} \mathrm{H}_{12} \mathrm{~N}_{2} \mathrm{O}_{2}$ erklärt werden kann. Eine kurze Mitteilung über diesen Befund haben wir in den Berichten der deutschen Chemischen Gesellschaft ${ }^{2}$ ) gemacht; im Folgenden beschreiben wir unsere inzwischen noch erweiterten Versuche ausführlicher und knüpfen an die Ergeb- nisse derselben einige Bemerkungen über die Constitution des Ornithins und des Arginins.

Darstellung von Ornithin aus Arginin.

E. Schulze und A. Likiernik extrahirten den bei der Zersetzung des Arginins entstandenen Harnstoff durch heissen Weingeist aus dem Syrup, welcher erhalten wurde, als sie die beim Kochen des Arginins mit Barytwasser entstandene Lösung durch Einleiten von Kohlensäure vom Baryt befreiten und sodann im Wasserbade eindunsteten. Nach dem Abgiessen des alkoholischen harnstoffhaltigen Extractes hinterblieb eine stark alkalisch reagirende Masse. Diese noch in unseren Händen befindliche Masse, welche sich sehr leicht in Wasser löste, bildete das Material für unseren ersten Versuch. In der Hoffnung, eine in derselben sich vorfindende Base als Benzoylverbindung isoliren zu können, wendeten wir auf sie das Baumann'sche Verfahren an, d. h. wir machten die wässerige Lösung jener Substanz mit Natronlauge alkalisch und setzten dann unter Umschütteln Benzoylchlorid in kleinen Portionen hinzu. Als der Flüssigkeit später Salzsäure zugefügt wurde, entstand eine starke milchige Trübung, welche sich bald in eine pflasterartige, am Boden und an den Wänden des Gefässes

1) Berichte der D. Chem. Gesellschaft, Bd. 10, S. 1925 u. Bd. 11, S. 406.

2) Bd. 30, S. 2879. 
fest anhaftende Masse verwandelte. Das so gewonnene Produkt war fast unlöslich in kochendem Wasser und liess sich daher durch wiederholte Behandlung mit diesem Lösungsmittel von beigemengter Benzoesäure befreien. In verdünnter Natronlauge oder Ammoniakflüssigkeit löste es sich auf und wurde aus dieser Lösung durch Säuren wieder gefällt. Es löste sich leicht in heissem Alkohol und krystallisirte daraus in kleinen Prismen. Wie aus den weiter unten gemachten Mittheilungen $\mathrm{zu}$ ersehen ist, erwies sich dieses Produkt als Ornithursäure $=\mathrm{C}_{19} \mathrm{H}_{20} \mathrm{~N}_{2} \mathrm{O}_{4}$. Diese Säure ist bekanntlich von M. Jaffé (loc. cit.) in den Excrementen von Hühnern, die mit Benzoesäure gefüttert worden waren, aufgefunden und als die Dibenzoylverbindung des Ornithins $=\mathrm{C}_{5} \mathrm{H}_{12} \mathrm{~N}_{2} \mathrm{O}_{2}$ erkannt worden.

Wir haben wiederholt sowohl freies Arginin wie Argininnitrat mit Barytwasser gekocht und die Flüssigkeit dann in der oben beschriebenen Weise behandelt; wir erhielten dabei stets Ornithursäure. Die Ausbeute an diesem Produkt war eine schwankende. Die beste Ausbeute erhielten wir, als wir die beschriebenen Operationen in folgender Weise austührten: Wir erhitzten eine Lösung von $9 \mathrm{gr}$. Argininnitrat und 16,5 gr. krystallisirtem Barythydrat in $540 \mathrm{ccm}$. Wasser 1 Stunde lang am Rückflusskühler zum Sieden, befreiten die noch heisse Flüssigkeit durch Einleiten von Kohlensäure vom Baryumhydroxyd und dunsteten sie sodann im Wasserbade zum Syrup ein. Aus diesem Syrup, welcher nur schwach gelb gefärbt war, wurde durch heissen Weingeist der Harnstoff extrahirt; den dabei verbliebenen Rückstand lösten wir in ca. $100 \mathrm{ccm}$. Wasser und fügten der Lösung unter häufigem Umschütteln abwechselnd Natronlauge und Benzoylchlorid in kleinen Antheilen $\left.\mathrm{zu}^{1}\right)$; im Ganzen wurden $70 \mathrm{ccm}$. 15\% ige Natronlauge und 15 gr. Benzoylchlorid zugesetzt. Nachdem die Flüssigkeit eine Zeit lang gestanden hatte, wurde sie mit Salzsäure angesäuert; dabei entstand eine starke weisse Ausscheidung, welche, nach-

1) Der Zusatz erfolgte in solcher Weise, dass die Flüssigkeit stets alkalisch blieb. 
dem sie durch Filtration von der Flüssigkeit getrennt war, zur Entfernung der beigemengten Benzoesäure wiederholt mit kochendem Wasser behandelt wurde. Das in Wasser Unlösliche lösten wir in heissem Weingeist. Die Lösung lieferte, nachdem sie durch Verdunsten auf ein geringeres Volumen gebracht worden war, $4,35 \mathrm{gr}$. reine krystallisirte Ornithursäure. Aus der Mutterlauge erhielten wir noch eine geringe Menge von Krystallen, welche zur Befreiung von etwa noch beigemengter Benzoesäure mit Aether behandelt wurden. Die Gesammtausbeute an Ornithursäure betrug 4,95 gr.

Im vorliegenden Versuch kam ein sehr gutes Benzoylchlorid-Präparat (frei von Benzaldehyd und Benzotrichlorid) zur Verwendung. In einem anderen Versuche, bei dessen Ausführung wir von der oben gegebenen Vorschrift nur wenig abwichen, aber ein weniger reines Benzoylchlorid-Präparat verwendeten, erhielten wir eine vor dem Umkrystallisiren aus Weingeist weniger reine Ornithursäure; doch war die Ausbeute fast eben so gross, wie im ersten Versuch; denn wir erhielten aus 18 gr. Argininnitrat ungefähr 9 gr. Ornithursäure.

Andere Versuche, in denen stärkeres Barytwasser angewendet und das Kochen am Rückflusskühler länger fortgesetzt wurde, lieferten beträchtlich niedrigere Ausbeuten an Ornithursäure.

Das Aussehen der in solcher Weise gewonnenen Ornithursäure und ihr Verhalten gegen Lösungsmittel entspricht den von Jaffé gemachten Angaben. Sie ist fast unlöslich in kochendem Wasser, ziemlich schwer löslich in kaltem, leicht löslich in kochendem Weingeist; aus der weingeistigen Lösung krystallisirt sie in kleinen farblosen Prismen. Der Schmelzpunkt der Ornithursäure liegt nach Jaffé bei $182^{\circ}$. Einige unserer Präparate zeigten den gleichen Schmelzpunkt; die durch mehrmaliges Umkrystallisiren aus Weingeist gereinigten Präparate schmolzen aber steț etwas höher, nämlich bei $184^{\circ}$. Die unter Verwendung von zwei Präparaten ${ }^{1}$ ) ausgeführten

1) Dieselben wurden, ehe sie zur Analyse verwendet wurden, bei $100^{\circ}$ getrocknet. 
Elementaranalysen lieferten Zahlen, welche der von Jaffé aufgestellten Formel $\mathrm{C}_{19} \mathrm{H}_{20} \mathrm{~N}_{2} \mathrm{O}_{4}$ entsprechen, wie folgende Angaben beweisen:

C- und H-Bestimmungen:

1) $0,2094 \mathrm{gr}$. Substanz gaben $0,5170 \mathrm{gr} . \mathrm{CO}_{\mathbf{8}}$ und $0,1197 \mathrm{gr} . \mathrm{H}_{8} \mathrm{O}$

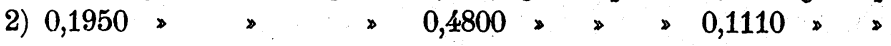

3) $0,1924>>0,4758>>0,1040$, ,

$\mathrm{N}$-Bestimmungen:

1) 0,2006 gr. Substanz gaben $16,2 \mathrm{ccm}$. feuchtes Stickstoffgas bei $20^{\circ}$ und $725 \mathrm{~mm}$. Druck.

2) 0,2046 gr. Substanz gaben $15,6 \mathrm{ccm}$. feuchtes Stickstoffgas bei $20^{\circ}$ und $720 \mathrm{~mm}$. Druck.

\begin{tabular}{cccccc} 
& Berechnet für & \multicolumn{4}{c}{ Gefunden } \\
\cline { 3 - 7 } & $\mathrm{C}_{18} \mathrm{H}_{80} \mathrm{~N}_{2} \mathrm{O}_{4}$ & & 2 & 3 & Mittel \\
$\mathrm{C}$ & 67,06 & 67,34 & 67,13 & 67,48 & $67,32 \%$ \\
$\mathrm{H}$ & 5,88 & 6,34 & 6,32 & 6,00 & 6,22 \\
$\mathrm{~N}$ & 8,24 & 8,76 & 8,25 & - & 8,51 \\
0 & 18,82 & - & - & - & -
\end{tabular}

Charakteristisch für die Ornithursäure ist nach Jaffé das Calciumsalz, welches sich krystallinisch ausscheidet, wenn man eine neutrale Lösung von ornithursaurem Ammonium mit Chlorcalcium versetzt und die klare Flüssigkeit dann erhitzt. Unser Produkt lieferte ein solches Salz, als wir es nach Jaffé's Vorschrift behandelten. Der Calciumgehalt dieses Salzes betrug $5,48 \%$, während die Theorie 5,57\% verlangt. ${ }^{1}$ )

Durch kurzes Kochen mit concentrirter Salzsäure wird die Ornithursäure nach Jaffé in Benzoesäure und Monobenzoylornithin $=\mathrm{C}_{12} \mathrm{H}_{16} \mathrm{~N}_{2} \mathrm{O}_{3}$ gespalten. Das letztere Produkt ist im Gegensatz zur Ornithursäure fast unlöslich in Alkohol, dagegen löslich in heissem Wasser; aus der Lösung krystallisirt es in farblosen, ausserordentlich feinen Nadeln, welche bei 225 $-230^{\circ}$ schmelzen. Unsere Ornithursäure lieferte bei ein- bis zweistündigem Kochen mit ca. 20\% produkt, welches im Aussehen und im Verhalten gegen Lösungsmittel den von Jaffé für das Monobenzoylornithin gemachten

1) Analytische Belege: 0,154 gr. des Calciumsalzes lieferten 0,0118 gr. $\mathrm{CaO}$. 
Angaben entsprach. Der Schmelzpunkt dieses Proakts war nicht constant; er schwankte von $225-240^{\circ}$.

Die im Vorigen gemachten Mittheilungen lasen kaum einen Zweifel daran übrig, dass der in der beschriebeen Weise von uns dargestellte Körper Ornithursäure oder Dienzoylornithin war; weitere Beweise für diese Annahme eben die Resultate, welche wir bei der Spaltung dieses Körpes durch concentrirte Salzsäure erhielten. Bei dieser Spaltug liefert die Ornithursäure nach Jaffé's Angaben neben einer richlichen Menge von Benzoesäure salzsaures Ornithin. Dieses Slz bleibt beim Eindunsten der von der ausgeschiedenen Bezoesäure durch Filtration getrennten Flüssigkeit als syrupöse, nah Zusatz von Alkohol krystallinisch werdende Masse zurück.

Wir führten das Erhitzen mit Salzsäure in solcbr Weise aus, dass wir die Ausbeute an den dabei entstehenen Produkten (Benzoesäure und salzsaures Ornithin) appoximativ bestimmen konnten. Die Ornithursäure wurde mit conentrirter Salzsäure in einem geräumigen Kolben 10 Stundenlang am Rückflusskühler erhitzt. Von der bei der Spaltung enttandenen Benzoesäure sublimirte ein Theil in die Kühlröhre hiein; wir brachten ihn mit Hülfe eines Glasstabs und der Spttzlasche in den Kolben zurück. Nach dem Erkalten wurde die auskrystallisirte Benzoesäure auf einem Filter gesamılt, das Filtrat sodann im Wasserbade auf ein geringes Volmen gebracht. Beim Erkalten schied sich wieder Benzoesure aus, welche mit der zuerst erhaltenen vereinigt, über Schrefelsäure getrocknet und dann gewogen wurde; die Ausbeute etrug im Durchschnitt 62\% der Ornithursäure. Jaffé erhielt aus der Ornithursäure 64,8\% Benzoesäure. Die Theorie verlang 71,8\%; es ist aber klar, dass das Aufsammeln des genanntenProdukts nicht ohne Verlust geschehen kann.

Das Filtrat von der Benzoesäure wurde unterWasserzusatz wiederholt eingedunstet, um die überschüssige jalzsäure und die in der Flüssigkeit noch vorhandenen Benzoeäurereste möglichst vollständig zu entfernen. Der dabei verblioene, nur schwach gefärbte Syrup, welcher das salzsaure Ornhin enthielt, wurde mit absolutem Alkohol übergossen. Er vewandelte 
sich in 1-2 Tagen in eine farblose Krystallmasse, welche nach dem Abgiessen der gelb gefärbten weingeistigen Flüssigkeit und Nachwaschen mit Alkohol im Exsiccator getrocknet wurde. Wie gross die Ausbeute war, ist aus folgenden Zahlen $\mathrm{zu}$ ersehen:

a) 4,0 gr. Ornithursäure lieferten 1,79 gr. krystallisirtes salzs. Ornithin

b) 3,8 > $>1,82$.

Aus diesen Zahlen berechnet sich, unter Berücksichtigung des durch Analyse bestimmten Salzsäuregehalts des Chlorhydrats, dass die Ausbeute an Ornithin im Durchschnitt 30,5\% der Ornithursäure betrug. Nach der Theorie sollen 100 Theile Ornithursäure 38,82 Theile Ornithin liefern. Es ist klar, dass auch die Gewinnung dieses Spaltungsprodukts der Ornithursäure nicht ohne Verlust geschehen kann; denn das salzsaure Ornithin ist nicht ganz unlöslich in Weingeist.

Das Chlorhydrat, welches Jaffé erhielt, als er die Ornthursäure in der beschriebenen Weise verarbeitete, war, nach. dem Ergebniss der Analyse, nach der Formel $\mathrm{C}_{5} \mathrm{H}_{12} \mathrm{~N}_{2} \mathrm{O}_{2}, 1_{1 / 2} \mathrm{HCl}$ zusammengesetzt (allerdings blieb die gefundene Stickstoffmenge etwas hinter der berechneten zurück). Die Krystalle, welche wir in der gleichen Weise erhielten, zeigten einen etwas. schwankenden Chlorgehalt. Bei Verarbeitung eines sehr reinen Ornithursäure-Präparats resultirte ein aus ziemlich grossen, strahlig gruppirten Prismen bestehendes Chlorhydrat von ganz: homogenem Aussehen, dessen Gehalt an Stickstoff und Chlor der Formel $\mathrm{C}_{5} \mathrm{H}_{12} \mathrm{~N}_{2} \mathrm{O}_{2}, 2 \mathrm{HCl}$ entsprach, wie aus folgenden Angaben $\mathrm{zu}$ schliessen ist:

1) 0,1200 gr. Subst. geb. 0,1674 gr. AgCl.

2) $0,1130, \quad>0,1570$,,

3) 0,2033 》 $\quad 25,2 \mathrm{ccm}$ feuchtes N-Gas bei $21^{\circ} \mathrm{u} .728 \mathrm{~mm}$. Druck

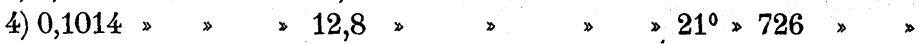

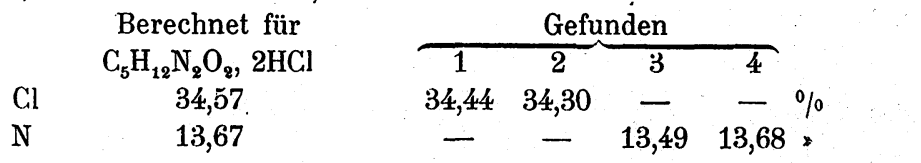

In anderen Versuchen erhielten wir Krystalle von geringerem Chlorgehalt (gef. 33,3, bezw. 32,5\% Cl). Von den bezüglichen Präparaten war wenigstens das eine zweifellos 
nicht homogen; es war deutlich zu sehen, dass es aus zwei verschiedenen Krystallarten bestand. Man darf vermuthen, dass in diesen Fällen dem Salz $\mathrm{C}_{5} \mathrm{H}_{12} \mathrm{~N}_{2} \mathrm{O}_{2}$, $2 \mathrm{HCl}$ das anderthalbfachsaure Salz (vergl. oben) oder das von Jaffé gleichfalls dargestellte Monochlorhydrat in gewisser Quantität beigemengt war. ${ }^{1}$ )

Das freie Ornithin konnte von Jaffé nicht krystallisirt erhalten werden. Auch uns ist dies nicht gelungen. Wir verwandelten, um die freie Base zu erhalten, das Chlorhydrat zunächst in das Sulfat, und zwar durch Umsetzung mit der berechneten Menge von Silbersulfat; das Sulfat zersetzten wir sodann durch die berechnete Quantität von Barythydrat. Die vom Baryumsulfat abfiltrirte Lösung der freien Base wurde in einem U-förmigen Glasrohr im $\mathrm{CO}_{2}$-freien Luftstrom (unter Herstellung eines partiellen Vacuums) eingedunstet. Wir erhielten einen Syrup, der sich allerdings mit einer festen Haut bedeckte, aber selbst nach dem Einbringen in eine Kältemischung nicht Krystalle bildete.

Das Nitrat der Base, dargestellt durch Neutralisation einer wässerigen Lösung der Base mit Salpetersäure, krystallisirte, gleich dem von Jaffé in entsprechender Weise dargestellten Salz $\mathrm{C}_{5} \mathrm{H}_{12} \mathrm{~N}_{2} \mathrm{O}_{2}, \mathrm{HNO}_{3}$, in Blättern. Es liess sich auch leicht zum Krystallisiren bringen, indem man seine nicht zu verdünnte, wässerige Lösung mit Alkohol und Aether vermischte. Auch diese Thatsache steht in Uebereinstimmung mit den von Jaffé gemachten Beobachtungen.

Aus den im Vorigen gemachten Mittheilungen ist mit Sicherheit zu schliessen, dass bei der Spaltung des Arginins durch Barytwasser Ornithin entsteht.

Das für unsere Versuche verwendete Arginin war theils aus Keimpflanzen von Lupinus luteus, theils durch Erhitzen von Eiweisssubstanzen mit Salzsäure dargestellt worden. $\mathrm{Ob}$ es der einen oder der anderen Quelle entstammte, war ohne

1) Aus einer Mittheilung F. Kutscher's (diese Zeitschrift, Bd. XXV, S. 199) geht hervor, dass auch das Lysindichlorid und das Histidindichlorid nur schwierig von einer den Formeln genau entsprechenden Zusammensetzung zu erhalten sind; meistens findet man bei ihnen etwas zu wenig Salzsäure. 
Einfluss auf das Resultat - ein neuer Beweis für die Identität der aus Keimpllanzen und der aus Eiweissstoffen gewonnenen Arginin-Präparate.

\section{Darstellung von Harnstoff aus Arginin.}

Eine Bestätigung der von E. Schulze und A. Likiernik (loc. cit.) über die Entstehung von Harnstoff aus Arginin gemachten Angabe liess sich leicht erhalten, indem wir den Syrup, welcher die bei der Einwirkung des Barythydrats auf Arginin entstandenen Produkte enthielt (vergl. oben), mit heissem Weingeist behandelten, den so erhaltenen Auszug eindunsteten und dem Verdampfungsrückstand concentrirte Salpetersäure zusetzten. Es schied sich Harnstoffnitrat aus, welches durch Abpressen zwischen Fliesspapier oder durch Aufstreichen auf eine Thonplatte von der Mutterlauge getrennt und sodann mit Baryumcarbonat und Wasser erhitzt wurde. Der beim Eindunsten der Flüssigkeit erhaltene Rückstand gab an heissen Weingeist Harnstoft $a b$, welcher aus der Lösung in dünnen Prismen krystallisirte.

\section{Ueber die Constitution des Ornithins.}

Auf Grund der bei Analyse der Salze des Ornithins, soẁie seiner Benzoylverbindungen erhaltenen Resultaţe hat Jaffé die Vermuthung ausgesprochen, dass diese Base eine Diamidovaleriansäure sei. Man kannte damals noch keine Diamidofettsäuren, mit denen man das Ornithin hätte vergleichen können. Inzwischen ist eine solche Verbindung, nämlich die Diamidopropionsäure, von E. Klebs ${ }^{1}$ ) synthetisch dargestellt worden. Dieser Base ist das Ornithin in vielen Punkten ähnlich. Zunächst ist darauf hinzuweisen, dass die Salze der Diamidopropionsäure ebenso wie die Salze des Ornithins in Wasser leicht. löslich, dagegen in Weingeist fast unlöslich sind und dass ihre concentrirten wässerigen Lösungen durch Zusatz von Weingeist

1) Inaugural-Dissertation, Tübingen 1894; diese Zeitschrift, Bd. XIX, S. 301. Die Arbeit ist im Hüfner'schen Laboratorium ausgeführt worden. 
meistens zum Krystallisiren gebracht werden. Ferner zeigt sich grosse Aehnlichkeit im Verhalten der freien Basen gegen Fällungsmittel. Nach den von uns angestellten Versuchen gibt eine verdünnte wässerige Lösung des Ornithins folgende Reactionen:

Mit Phosphorwolframsäure starke weisse Fällung

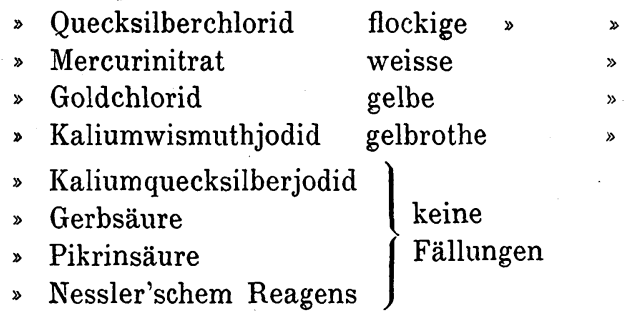

Das gleiche Verhalten gegen die im Vorigen genannten Reagentien zeigt nach den Versuchen von Klebs die freie Diamidopropionsäure in verdünnter wässeriger Lösung; doch wird sie auch durch Phosphormolybdänsäure gefällt, während dieses Reagens mit Ornithin keine Fällung gab.

Die wässerigen Lösungen der. Diamidopropionsäure und des Ornithins besitzen stark alkalische Reaction; sie lösen gelbes Quecksilberoxyd und Kupferoxydhydrat; die so entstandenen. Flüssigkeiten besitzen noch in sehr starker Verdünnung alkalische Reaction. Die Lösungen der Kupferverbindungen sind intensiv blau gefärbt. Ein Unterschied aber besteht darin, dass sowohl die Kupfer- wie die Quecksilberverbindung der Diamidopropionsäure sich in Krystallform überführen lässt, indem man die Lösungen zum Syrup eindunstet und letzteren mit Weingeist übergiesst; bei den entsprechenden Ornithinverbindungen gelanr uns dies nicht.

Wie das Ornithin gibt auch die Diamidopropionsäure na den Versuchen von Klebs eine Dibenzoylverbindung, welc wenig löslich in Wasser, dagegen leicht löslich in Weingeist $\mathrm{i}$

Die Uebereinstimmung, welche in vielen Punkten zwisch der Diamidopropionsäure und dem Ornithin sich zeigt, mac es sehr wahrscheinlich, dass auch das letztere eine Diamid fettsäure ist; dasselbe kann aber in diesem Falle, nach d, 
beei der Analyse seiner Salze etc. erhaltenen Resultaten, nur eiine Diamidovaleriansäure sein. ${ }^{1}$ )

Ist das Ornithin eine Diamidovaleriansäure, so darf man errwarten, dass es durch salpetrige Säure nach der Gleichung

$$
\mathrm{C}_{5} \mathrm{H}_{12} \mathrm{~N}_{2} \mathrm{O}_{2}+2\left(\mathrm{HNO}_{2}\right)=\mathrm{C}_{5} \mathrm{H}_{10} \mathrm{O}_{4}+4 \mathrm{~N}+2\left(\mathrm{H}_{2} \mathrm{O}\right)
$$

zersetzt wird; 132 Gewichtstheile Ornithin müssen also mit überschüssiger salpetriger Sắure 56 Gewichtstheile freien Sticksitoffs liefern. Um zu prüfen, ob dies der Fall sei, wendeten wir auf das Nitrat des Ornithins das Verfahren von R. Sachsse und WW. Kormann ${ }^{2}$ ) an, welches es gestattet, die bei Zersetzung eiines Amids durch salpetrige Säure entwickelte Stickstoffmenge quantitativ zu bestimmen. Wir erhielten folgende Zahlen:

a) $0,100 \mathrm{gr}$. Ornithinnitrat gab $28 \mathrm{ccm}$. Gas bei 220 und $726 \mathrm{~mm}$. Druck $=0,03026$ gr. $\mathrm{N}$.

b) 0,100 gr. Ornithinnitrat gab 27,5 ccm. Gas bei 220 und $728 \mathrm{~mm}$ Druck $=0,02981$ gr. $\mathrm{N}$.

Da in 0,100 gr. des Nitrats 0,6769 gr. Ornithin enthalten sind, so haben in den vorstehenden Versuchen 132 Theile Ornithin mit salpetriger Säure im Durchschnitt 58,6 Theile Stickstoff entwickelt, also noch etwas mehr, als der obigen Cưleichung entspricht. ${ }^{3}$ ) Dieses Resultat führt zu der Annahme, dlass das Ornithin zwei durch salpetrige Säure zersetzbare $\mathrm{NVH}_{2}$-Gruppen enthält.

Sind diese $\mathrm{NH}_{2}$-Gruppen an zwei benachbarte oder an zwwei in der Kette voneinander entfernt stehende Kohlenstoffatome gebunden? Ein Weg zur Entscheidung dieser Frage schien sich aus der Thatsache zu ergeben, dass aromatische Orthodiamine mit Orthodiketonen schwer lösliche Condensationsrodukte (Chinoxalinderivate) geben. Wir brachten eine concenrirte Lösung von Ornithinacetat mit einer essigsauren Lösung von 'henanthrenchinon zusammen, erhielten dabei aber keine

1) Auch Klebs (loc. cit.) sieht das Ornithin als eine Diamidoaleriansäure an.

2) Landwirthschaftliche Versuchsstationen, Bd. 17, S. 321.

3) Das in den obigen Versuchen von uns in Anwendung gebrachte Jerfahren liefert nach den sowohl von Sachsse und Kormann (loc. cit.), vie von Anderen gemachten Angaben in der Regel etwas zu hohe Zahlen. 
Ausscheidung. Dies scheint zu der Schlussfolgerung zu führen, dass im Ornithinmolekül die beiden $\mathrm{NH}_{2}$-Gruppen nicht mit benachbarten Kohlenstoffatomen verbunden . sind. ${ }^{1}$ )

Die Ornithinquantität, die wir zur Zeit zur Verfügung hatten, war leider nicht gross genug, um die bei Zersetzung dieser Base durch salpetrige Säure wahrscheinlich entstehende Oxysäure untersuchen zu können.

\section{Ueber die Constitution des Arginins.}

Es ist nachgewiesen, dass bei Zersetzung des Arginins durch Barytwasser Harnstoff entsteht; diese Thatsache lässt es als möglich erscheinen, dass das Arginin ein Derivat des Guanidins ist. Da nun bei dieser Zersetzung neben Harnstoff Ornithin sich bildet und da letzteres als Diamidovaleriansäure anzusehen ist, so darf es vielleicht für wahrscheinlich erklärt werden, dass dem Arginin die Constitutionsformel

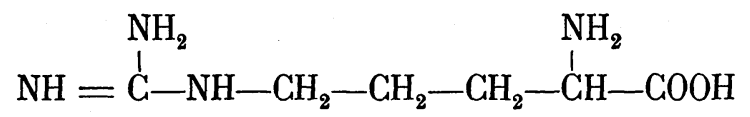

zukommt. ${ }^{2}$ ) Das Arginin würde in diesem Falle ein Körper sein, welcher in der Structur dem Glycocyamin und mit dem Kreatin ähnlich ist. Die Bildung von Harnstoff und Ornithin. aus Arginin könnte nach folgender Gleichung stattfinden:

$$
\mathrm{C}_{6} \mathrm{H}_{14} \mathrm{~N}_{4} \mathrm{O}_{2}+\mathrm{H}_{2} \mathrm{O}=\underbrace{\mathrm{CO}}_{\mathrm{NH}_{2}}+\mathrm{C}_{5} \mathrm{H}_{12} \mathrm{~N}_{2} \mathrm{O}_{2}
$$

Freilich betrug in unseren Versuchen die Ausbeute an Ornithin höchstens $40 \%$ der aus vorstehender Gleichung sich

1) Allerdings ist, so viel wir wissen, bis jetzt noch nicht geprüft worden, wie sich aliphatische Körper bei der obigen Reaction verhalten.

2) Nach einer mündlichen Mittheilung, welche der eine von uns (E. S.) - im Sommer 1897 von dem leider bald darauf aus dem Leben abberufenen E. Dre chsel erhielt, theilte dieser Forscher die oben wiedergegebene Anschauung über die Constitution des Arginins. Nach einer in dieser Zeitschrift, Bd. XXV, S. 189 gemachten Bemerkung würde dagegen A. Kossel einer Structurformel den Vorzug geben, in welcher die 6 Kohlenstoffatome miteinander verbunden sind. 
berechnenden Quantität ${ }^{1}$ ) es ist aber möglich, dass hier, wie beim Kreatin, ${ }^{2}$ ) die Zersetzung durch Barytwasser in zwei verschiedenen Richtungen verläuft. Dafür scheint auch die Thatsache zu sprechen, dass beim Kochen mit stärkerem Barỵtwasser das Arginin eine beträchtlich geringere. Ornithinmenge lieferte, als bei Befolgung der oben von uns mitgetheilten Vorschrift. ${ }^{3}$ )

Ist diese Annahme richtig, so muss bei Zersetzung des Arginins durch Barytwasser neben Harnstoff und Ornithin noch eine andere organische Stickstoffverbindung entstehen; was dies aber für eine Verbindung ist, vermögen wir zur Zeit nicht zu sagen. Das Filtrat von der Ornithursäure (vergl. oben) gab mit Phosphorwolframsäure einen starken Niederschlag. Die bei Zerlegung dieses Niederschlags durch Barytwasser entstandene Lösung gab nach der Neutralisation eine starke Fällung mit Mercurinitrat; doch haben wir bis jetzt aus diesen Niederschlägen einen wohlcharakterisirten Körper nicht zu isoliren vermocht. Dass im Filtrat von der Ornithursäure noch unverändertes Arginin sich vorfand, ist nicht anzunehmen; denn wir vermochten daraus, nach nochmaligem Kochen mit Barytwasser, keine Ornithursäure mehr zu gewinnen.

Bei Erörterungen, deren Gegenstand die Constitution des Arginins ist, verdient auch der Umstand berücksichtigt $\mathrm{zu}$ werden, dass beim Zusammenbringen des Arginins mit salpetriger Säure im Durchschnitt nur $1 / 8$, in allen Fällen aber weniger als die Hälfte der Stickstoffmenge, die in der genannten Base sich findet, in Freiheit gesetzt wurde. ${ }^{4}$ )

1) Die Maximalausbeute an Ornithursäure, die wir aus Arginin nach der oben von uns mitgetheilten Vorschrift erhielten, entspricht einer Ausbeute an Ornithin, welche nahezu $40 \%$ der nach obiger Gleichung berechneten Menge beträgt.

2) Bekanntlich liefert das Kreatin beim Erhitzen mit Barytwasser nicht nur Harnstoff und Sarkosin, sondern auch Methylhydantoin.

3) Allerdings würde diese Erscheinung auch ihre Erklärung haben, wenn man annimmt, dass das Ornithin beim Erhitzen mit Barytwasser sich langsam zersetzt.

4) Nach den von E. Schulze und E. Steiger (diese Zeitschrift, Bd. XI, S. 59) angestellten Versuchen. Die bezüglichen Bestimmungen gaben jedoch stark schwankende Resultate. 
Besitzt das Arginin eine Structur, welche ler obigen Formel entspricht, so wird es vielleicht möglich ein, durch Einwirkung von Cyanamid auf Ornithin das Aginin synthetisch darzustellen. Wir hoffen diese Frage auf dem Wege des Experiments entscheiden zu können, sind aber bis jetzt, da uns das erforderliche Material nur in beschräniter Menge zur Verfügung stand, über Vorversuche nicht hinaussekommen. 